\title{
Lab-level and low-cost fabrication technique for polymer based micro-optical elements and holographic structures
}

\author{
Maik Rahlves ${ }^{a}$, Maher Rezem ${ }^{a}$, Christian $\mathrm{Kelb}^{a}$, Kristian Boroz $^{a}$, Dina Gödeke ${ }^{a}$, Sebastian \\ Schlangen $^{a}$, Eduard Reithmeier ${ }^{b}$, Bernhard Roth ${ }^{a}$ \\ ${ }^{a}$ Hannover Center of Optical Technologies, Leibniz Universität Hannover, Nienburger Strasse \\ 17, Hannover, Germany; \\ ${ }^{b}$ Institute of Measurement and Automatic Control, Leibniz Universität Hannover, Nienburger \\ Strasse 17, Hannover, Germany
}

\begin{abstract}
Polymer based diffractive optical elements have gained increasing interest due to their potential to be used in various applications such as illumination technology, micro optics and holography. We present a novel production process to fabricate polymer based diffractive optical elements and holograms. The process is based on maskless lithography, which is used to fabricate optical elements in photoresist. We discuss several lab-made lithography setups based on digital mirror devices and liquid crystal devices with respect to light efficiency, resolution and contrast. The whole optical setup is designed with an emphasis on low-cost setups, which can be easily implemented in an optical research lab. In a subsequent step, a copy of the microstructures is easily replicated into optical polymeric materials by means of a soft stamp hot embossing process step. The soft stamp is made from Polydimethylsiloxan, which is coated onto the microstructure in resist. The hot embossing process is carried out by a self-made and low-cost hot embossing machine. We present confocal topography measurements to quantify the replication accuracy of the process and demonstrate diffractive optical elements and holographic structures, which were fabricated using the process presented.
\end{abstract}

Keywords: Polymer optics, diffractive optics, holography, maskless lithography, hot embossing

\section{INTRODUCTION}

Holograms and diffractive optical elements (DOE) are widely used in optics for applications ranging from beam shaping to imaging application. ${ }^{1}$ Especially polymers as material for diffractive microstructures offer advantages such as easy and low-cost production techniques compared to glass or other common materials. ${ }^{1-3}$ For a high throughput and low-cost production of polymer based DOE, micro-injection molding is one of the most common replication processes. It is based on an extruder which injects a fused polymer into a micro-mold. The mold is cooled down subsequently and the polymer DOE is released. If a high throughput is not required, e.g., for small custom made series of DOEs, hot embossing offers an alternative as replication technique. ${ }^{3}$ For hot embossing, a master stamp and a polymer substrate are heated well above the glass transition temperature of the polymer. An embossing force is applied to the stamp, which is embossed into the deformable polymer. The DOE is released from the stamp after cooling below the glass transition temperature. Both replication processes require highly accurate molds and stamps, which meet optical requirements in terms of form accuracy and surface roughness. Therefore, the most expensive and time consuming part of the production process is the stamp and

Further author information: (Send correspondence to M.Ra.)

M.Ra.: E-mail: maik.rahlves@hot.uni-hannover.de, Telephone: 4951176217901

M.Re.: E-mail: maher.rezem@hot.uni-hannover.de, Telephone: 4951176217303

C.K..: E-mail: christian.kelb@hot.uni-hannover.de, Telephone: 4951176217943

K.B..: E-mail: kristian.boroz@hot.uni-hannover.de, Telephone: 4951176217908

D.G..: E-mail: dina.goedeke@hot.uni-hannover.de, Telephone: 4951176217908

S.S..: E-mail: sebastian.schlangen@hot.uni-hannover.de, Telephone: 4951176217977

E.R..: E-mail: sekretariat@imr.uni-hannover.de, Telephone: 495117623334

B.R..: E-mail: bernhard.roth@hot.uni-hannover.de, Telephone: 4951176217907

Holography: Advances and Modern Trends IV, edited by Miroslav Hrabovský,

John T. Sheridan, Antonio Fimia, Proc. of SPIE Vol. 9508, 95080H · C 2015

SPIE · CCC code: $0277-786 \mathrm{X} / 15 / \$ 18 \cdot$ doi: $10.1117 / 12.2178890$

Proc. of SPIE Vol. $950895080 \mathrm{H}-1$ 


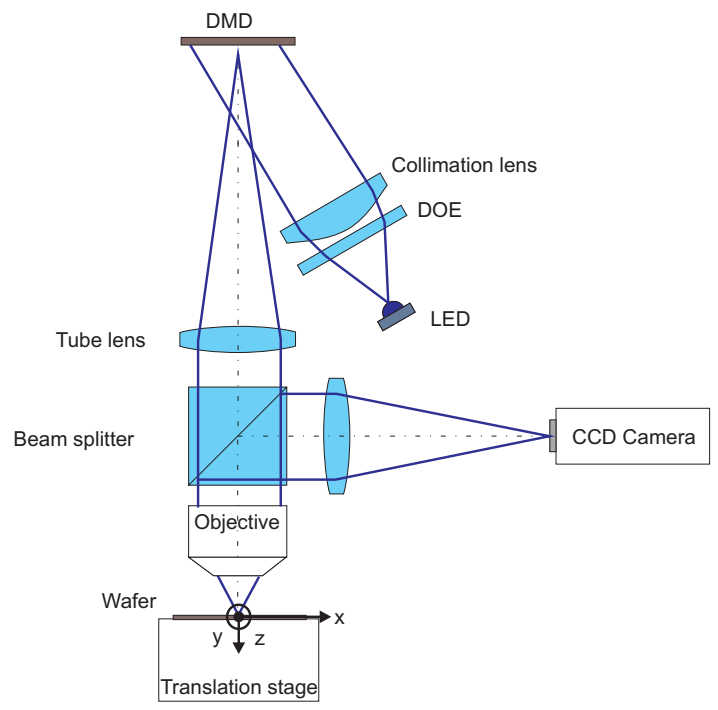

Figure 1. Experimental setup of the maskless lithography unit using a DMD.

mold production itself, which is often based on ultra precision milling and turning or various etching techniques known from microsystem technology. ${ }^{1,3,4}$ As an alternative, the stamp geometry can be directly written into photosensitive resist by direct laser writing or maskless lithography. ${ }^{5-7}$ Since photoresist is not suitable as stamp material, methods were developed to create a copy of the microstructured resist in PDMS or nickel, which is used as stamp for hot embossing or injection molding subsequently. ${ }^{6-8}$ Also direct embossing of holographic microstructures in sheet metals using maskless lithography to generate arbitrary macro and micro geometries was reported. ${ }^{8}$

In this work, we present two optical setups for maskless lithography to generate a stamp geometry in photoresist. The first setup is based on digital mirror devices (DMD) and the second on liquid crystal displays (LCD) to create an image of the desired microstructure, which is transferred to the photoresist using a simple microscope setup. After development of the resist, we fabricated a PDMS copy, which was used as stamp for hot embossing. We employed a commercially available hot embossing machine as well as a self-made and low cost device to replica the stamp in polymer.

\section{PRODUCTION PROCESS}

To fabricate the hot embossing stamp, we created an inverse of the desired microstructure of the DOE in photoresist (Shipley 1814) by means of two different self-made maskless lithography setups. The first setup is based on a liquid crystal display and the second on a digital mirror device as explained in detail in this section:

\subsection{Production of Master Stamp Using DMD Based Maskless Lithography}

The lithography setup which is based on a DMD is illustrated in Fig. 1. A DMD consists of an array of micro mirrors, which deflect incoming light into two different directions. Each pixel can be addressed separately to generate an image of the master stamp layout at a resolution of 1024 times 768 pixel. A DMD device (Vialux ALP 4.1), which is illuminated by a high power light emitting diode (LED) with a center wavelength of $435 \mathrm{~nm}$ and a maximum optical outpout power of $350 \mathrm{~mW}$ generates an image of the DOE microstructure. The image is demagnified by a microscope setup (Zeiss) and projected onto a silicon substrate coated with photoresist. Additionally, the substrate is placed on piezo-electric translation stage (Smaract), which can move the substrate laterally with respect to the microscope and enables us to perform a stitching process to obtain a larger structurable field size compared to the field of view of the microscope setup. We achieved a maximal structurable field size of $5 \mathrm{~cm}$ times $5 \mathrm{~cm}$ at a resolution of $1.35 \mu \mathrm{m}$ feature size when using a microscope lens with a numerical aperture of 0.3 (Zeiss Epiplan 10x). To generate a sharp image of the DMD on the silicon substrate, 


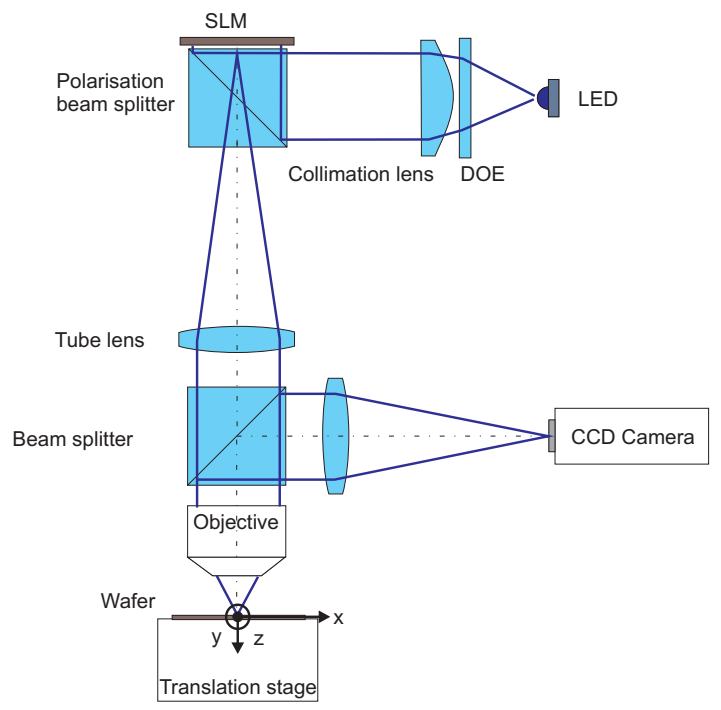

Figure 2. Experimental setup of the maskless lithography unit using a SLM.

the image reflected by the silicon wafer is imaged onto a CCD camera (Stemmer Imaging). By shifting the substrate along the optical axis of the microscope lens manually using a mechanical translation stage (Thorlabs), the vertical position of the substrate where a sharp image is generated was found.

Prior to the lithography process step, we utilized a spin coating process to obtain a homogeneous layer of photoresist with a thickness of approximately $500 \mathrm{~nm}$ on a substrate. A standard 3 inch silicon wafer with a thickness of $500 \mu \mathrm{m}$ served as substrate material. After spin coating, a prebake at $115^{\circ} \mathrm{C}$ was applied for $60 \mathrm{~s}$. As diffractive structure, we chose a simple grating structure with approximately $3 \mu \mathrm{m}$ pitch and a macro structure showing a logo of our project partner.

\subsection{Production of Master Stamp Using LCD Based Maskless Lithography}

An alternative setup to generate an image of the master stamp geometry is based on a LCD display and is shown in Fig. 2. The basic components such as microscope translation stages and light source are the same as in the DMD based setup shown in Fig. 1. However, for image formation, the collimated light beam of the LED is polarized by a polarizing beam splitter and directed onto the LCD display (Holoeye). The LCD display consists of 1980 times 1024 single pixels, which turn the polarization state of the incoming light. A mirror inside the display reflects the light back into the microscope passing through the polarization beam splitter again. As a consequence, the intensity of each pixel changes depending on the polarization state of the light and, hence, an intensity distribution is generated.

\subsection{Replication of Master Stamp by Hot Embossing}

In the subsequent process step shown in Fig 3, we transferred the topography of the master stamp in Polydimethylsiloxane (PDMS), which serves as a hot embossing stamp to obtain the final DOE in polymer. First, we coated a few millimeter thin layer of PDMS on the microstructured surface of the silicon substrate and cured the PDMS for 48 hours at room temperature.

As material for DOE production, we chose Polymethylmethacrylate (PMMA), which is a thermoplastic polymer often used for hot embossing. The glass transition temperature of PMMA is $T_{G}=105^{\circ} \mathrm{C}$. For replication, we utilized two different hot embossing devices, a commercially available HEX03 (Jenoptik) and a self-made hot embossing maschine shown in Fig. 4. The advantages of the latter device are low acquisition costs but it provides only a limited capability to control the embossing force. Our self-made hot embossing machine consists of a tool holder and a substrate holder. Both are mounted on two $200 \mathrm{~W}$ heating plates, which are monitored by PT100 thermistors. Each thermistor is connected to a control unit, which sets the heating level to a preset temperature 


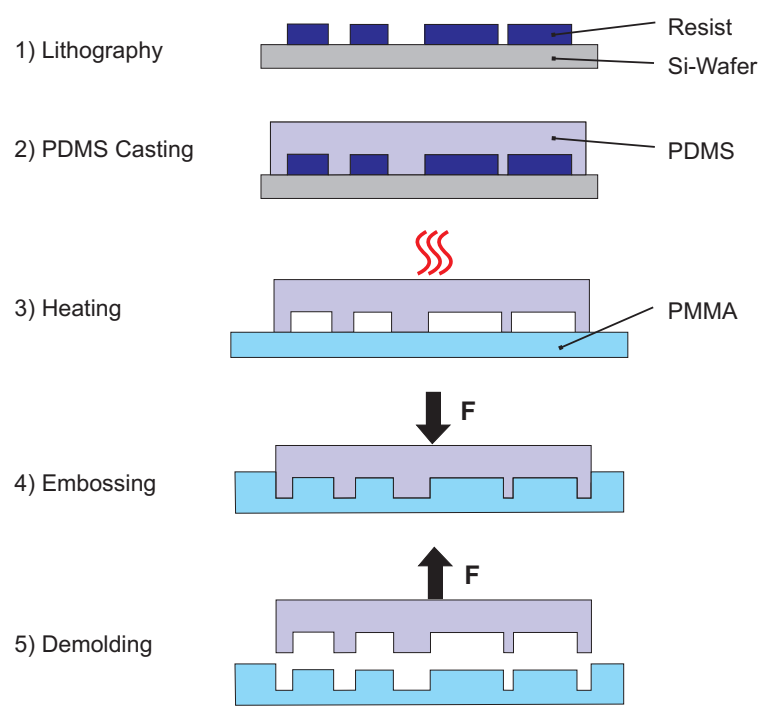

Figure 3. Replication process for master stamp replication into PMMA using soft stamp hot embossing.

value. The embossing force is applied manually using a hydrostatic pressure pump. To achieve a steady relative movement as well as to provide negligible tilt error between tool and substrate holder, the substrate holder is guided by guiding rods during the embossing cycle.

For comparison, we conducted two embossing runs using our self-made as well as the commercial hot embossing machine. The first run was carried out in the commercial device. Both, PDMS tool and PMMA substrate of $500 \mu \mathrm{m}$ thickness were heated to an embossing temperature of $120^{\circ} \mathrm{C}$ and an embossing pressure of $6.2 \mathrm{kPa}$ was applied for 5 min. The PMMA DOE was removed manually from the PDMS after cooling down to an release temperature of $40^{\circ} \mathrm{C}$. The second embossing run was performed in the self-made hot embossing machine, where we also used a hot embossing temperature of $120^{\circ} \mathrm{C}$. However, since our device does not include a pressure sensor, the exact embossing pressure could not be determined.

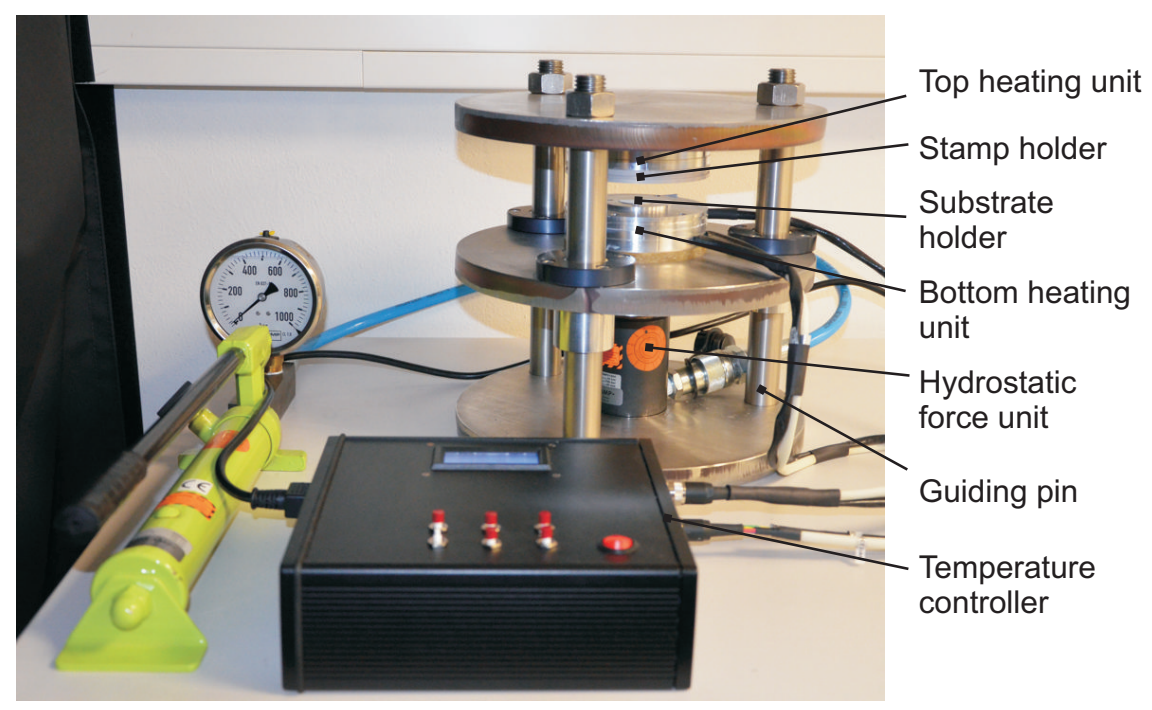

Figure 4. Lab-level and low-cost hot embossing maschine. 


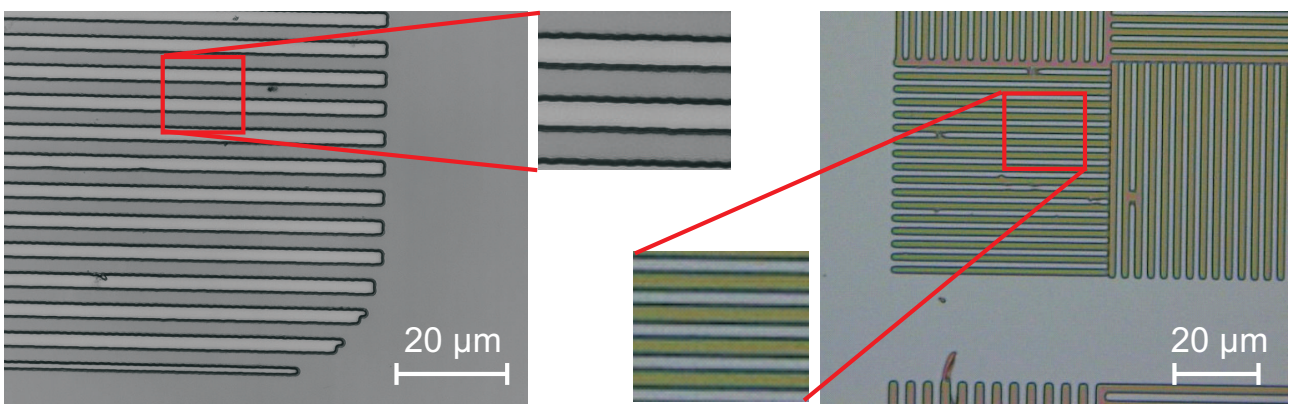

Figure 5. Microscope images of master stamps created in photoresist using the DMD based setup (left) and the LCD based setup (right).
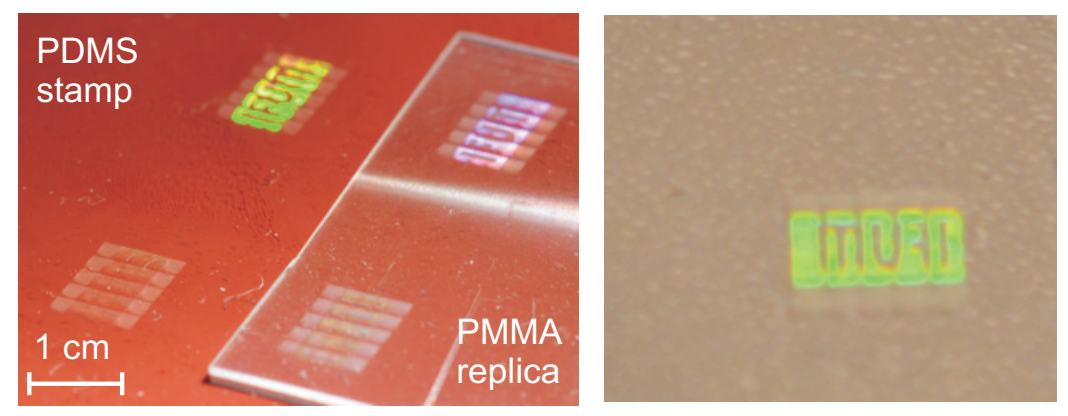

Figure 6. Large area diffractive structure on PDMS stamp and corresponding PMMA replica fabricated using the HEX03 (left) and the self-made hot embossing machine (right).

\section{RESULTS}

\subsection{Master Stamp Fabrication by DMD and LCD based Maskless Lithography}

Fig. 5 shows microscope images of the master stamps, which were created in photoresist utilizing the DMD and the LCD based setups. Both structures consist of linear diffraction gratings with period lengths of $5.4 \mu \mathrm{m}$ and $3 \mu \mathrm{m}$, respectively. However, the pattern generated using a DMD shows periodical defects at the side walls of the linear structures, which are apparent in the enlarged microscope image in Fig. 5. In contrast, the structures generated using a LCD show a significantly smaller line edge roughness. The roughness in the first image is due to a larger pixel size of $13.8 \mu \mathrm{m}$ of the DMD in comparison to the LCD, which exhibits a pixel pitch of $8 \mu \mathrm{m}$. When using a microscope lens with a numerical aperture of 0.3 , the theoretical resolution of the setup is $884 \mathrm{~nm}$ on the wafer, which corresponds to resolved pixel of $8.8 \mu \mathrm{m}$ in the display plane of the setup when considering a magnification of 10 of the microscope lens. Hence, the setup resolves the micro mirrors but not the single liquid crystal elements. This effects weakens when using objectives with higher numerical apertures. When comparing the results of both setups, we experienced that much more effort has to be made when designing suitable collimation optics for DMD compared to LCD based setups. Using an LCD, only a homogenous light distribution has to be generated to yield an uniform development of all microstructures throughout the entire field of view of the setup. For DMD illumination, we do not only have to consider a homogeneous light distribution but also that each light ray reflected by a micro mirror needs to be perfectly parallel to the optical axis of the microscope setup. A deviation leads to a blurred image of the microstructure and, hence, to significant decrease in the smallest achievable feature size of the setup. On the other hand, most DMDs, in contrast to common LCDs, can be used in combination with ultraviolet light sources, which allows for a larger variety of photoresists to be used. In addition, DMDs display smaller light absorption and higher light intensities are feasible which reduces the exposure time. In our case, exposure cycles for a single image were $7 \mathrm{~min}$ and $10 \mathrm{~s}$ using the LCD and DMD setups, respectively. 


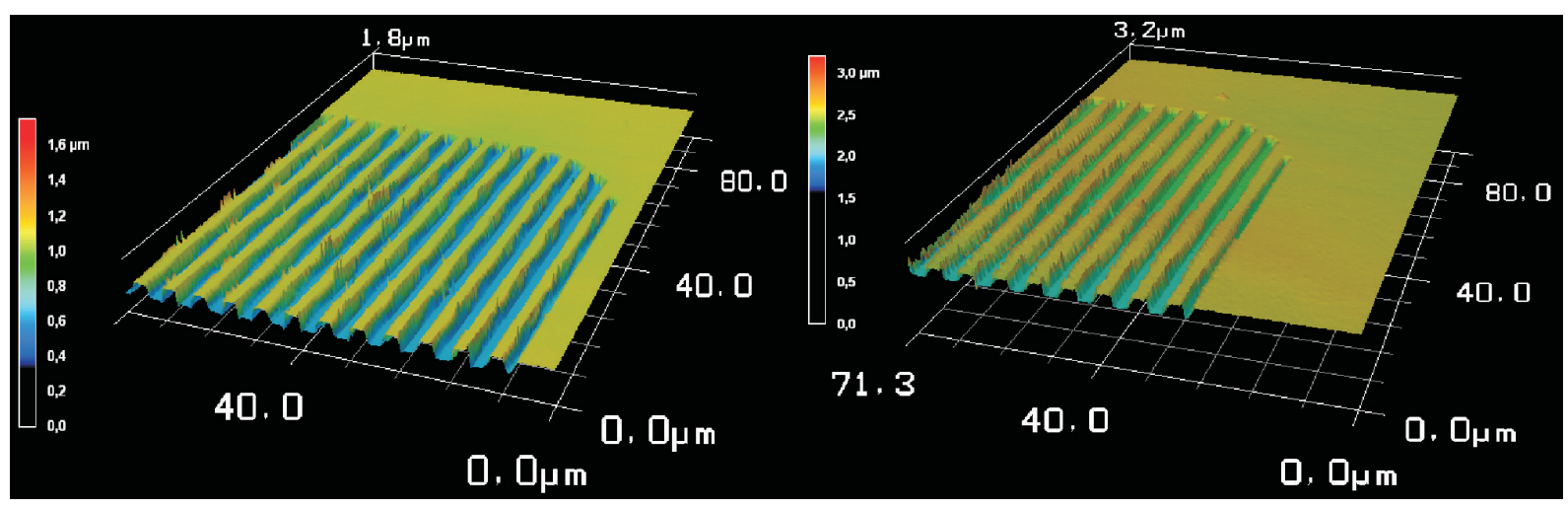

Figure 7. Confocal topography measurements of a stamp in photoresist on silicon substrate (left) and the PMMA replica (right).

\subsection{Hot embossing results}

A photograph of the PDMS copy obtained from the master stamp fabricated using the DMD based setup as well as a hot embossed PMMA replica utilizing the commercial HEX03 hot embossing machine are shown in Fig. 6 (left). A DOE fabricated by our self-made hot embossing machine in PMMA is shown in Fig. 6 (right). Both embossing results show a logo of our project partner as macro-geometry and a diffraction grating with $5.2 \mu \mathrm{m}$ pitch is incorporated additionally inside the logo, which leads to light diffraction as obvious in Fig. 6 . We carried out confocal topography measurements to quantify the error between master stamp, PDMS soft stamp, and PMMA replica. We found that in general, the micro structures are well replicated. The measured periods are $5.22 \mu \mathrm{m}, 5.32 \mu \mathrm{m}$, and $5.47 \mu \mathrm{m}$ and the measured profile heights are $492 \mathrm{~nm}, 431 \mathrm{~nm}$, and $471 \mathrm{~nm}$ of the master stamp, the PDMS stamp, and the PMMA replica fabricated using the HEX03, respectively. The increase in period length is caused by an expansion of the PDMS and PMMA during hot embossing due to the embossing force and polymer yielding. For comparison, Fig. 7 shows three-dimensional confocal microscope measurements of master stamp and PMMA replica. The measured topography shows the same section on the sample as in Fig. 6.

\section{CONCLUSION}

We introduced a process chain for low-cost production and replication of polymer based diffractive optical elements and holographic structures. The process chain relies on an initial lithographic process step, where a master is fabricated by means of maskless lithography. We presented two different optical setups for maskless lithography based on a digital mirror device (DMD) and a liquid crystal display (LCD). Our experimental results show that even if the optical setup and, especially, the optical design of the light source is less complicated using the liquid crystal display, the setup utilizing a digital mirror provides advantages regarding process time and the feasibility to operate at a shorter wavelength. In a subsequent step, we transferred the micro- and macro geometry of the fabricated master stamp into a PDMS copy, which was used for soft stamp hot embossing. We also presented hot embossing results of diffractive structure obtained by using a self-made and low-cost hot embossing machine. The casting quality of the results were compared to embossing results obtained using a professional hot embossing machine. No significant deviation in quality was observed. Hence, the presented process chain enables us to fabricate diffractive optical elements at high optical quality on a low-cost and lab-level basis. Our results will also be used in future work to fabricate more complex optical structures such as optical waveguide structures and sensors.

\section{Acknowledgments}

Parts of this work were funded by the German Research Foundation (DFG) in the framework of the collaborative research center PlanOS and the German Federation of Industrial Research Associations (AiF) under grant EFB ZN 500. 


\section{REFERENCES}

[1] Herzig, H., ed., [Micro-optics - Elements, Systems and Applications], Taylor \& Francis (1997).

[2] Bäumer, S., ed., [Handbook of Plastic Optics], (Wiley-VCH (2005).

[3] Worgull, M., [Hot Embossing: Theory and Technology of Microreplication], William Andrew (2009).

[4] Luo, X., Cheng, K., Webb, D., and Wardle, F., "Design of ultraprecision machine tools with applications to manufacture of miniature and micro components," J. Mater. Process. Technol. 167(2-3), 515-528 (2005).

[5] Gale, M. T., Rossi, M., Pedersen, J., and Schuetz, H., "Fabrication of continuous-relief micro-optical elements by direct laser writing in photoresists," Opt. Eng. 33(11), 3556-3566 (1994).

[6] Erdmann, L. H., Deparnay, A., Wirth, F., and Brunner, R., "Mems-based lithography for the fabrication of micro-optical components," in [Proc. SPIE 5347], 79-84 (2004).

[7] Rahlves, M., Rezem, M., Boroz, K., Schlangen, S., Reithmeier, E., and Roth, B., "A flexible, fast, and low-cost production process for polymer based diffractive optics," Opt. Express (2015).

[8] Behrens, B.-A., Krimm, R., Jocker, J., Reithmeier, E., Roth, B., and Rahlves, M., "Method to emboss holograms into the surface of sheet metals," Key Eng. Mater. 549, 125-132 (2013). 